\title{
CO-JP-7
}

\section{A Layout Design Optimization Method for Multi-robot Assembly Systems*}

\author{
Issei Suemitsu**, Takayuki Yamada**, Kazuhiro Izui**, Shinji Nishiwaki**, \\ Akio Noda***, Tatsuya Nagatani*** \\ **Department of Mechanical Engineering and Science, Kyoto University, \\ Yoshida-honmachi, Sakyo-ku, Kyoto 606-8501, Japan, \\ Email:suemitsu.issei.66a@st.kyoto-u.ac.jp \\ ***Mitsubishi Electric Corporation, Tsukaguchi-honmachi 8-1-1, Amagasaki, Hyogo, Japan, \\ noda.akio@dh.mitsubishielectric.co.jp
}

\begin{abstract}
This paper proposes a conceptual layout design method for robot cellular manufacturing systems (RCMS) using a multi-objective optimization. First, the design criteria for RCMS layouts are clarified and the objective functions are formulated. Next, layout design candidates are represented using a sequence-pair scheme with dummy components in order to avoid interference between assembly system components. A multi-objective genetic algorithm is then used to obtain Pareto optimal solutions for the layout optimization problems. Finally, several numerical examples are provided to illustrate the effectiveness and usefulness of the proposed method.
\end{abstract}

Key words: robot cell, assembly layout, design optimization, genetic algorithm

\section{Introduction}

Robotic cellular manufacturing systems (RCMS) have drawn attention due to their applicability in high-mix and low-volume production scenarios. RCMS is a new type of manufacturing system in which one or more flexible robots carry out a large number of assembly operations that would be performed by human workers in conventional cellular manufacturing systems.

When compared with conventional human cellular manufacturing systems, RCMS are seen to offer similar advantages, such as reduction of material flow distances and local inventory. However, although reduced operation costs due to automation of the manufacturing systems can be achieved by introducing RCMS, the design of assembly operations and robot teaching can become quite difficult and time-consuming when launching new manufacturing systems, due to the complexity of the dynamic nature of robot movements and the need to consider cooperative tasks.

The layout design stage is one of the most important stages when seeking to build efficient RCMS. The layout design stage is an upstream stage of the manufacturing system design process, and decisions made then exert considerable influence on the detailed design of robot motion planning and the teaching process that is later carried out.

Therefore, skillful decision-making during the layout design stage is essential, in order to minimize design changes during the detailed design stage and teaching process, and to enhance the efficiency and reliability of the manufacturing system when deployed. With this background in mind, certain optimization techniques have been applied to assist the layout design of RCMS [1] [2]. Unfortunately, these methods typically require explicit constraint handling regarding component overlapping, since component coordinates are handled as design variables, and this implementation obstructs global searching of the solution space. Layout problems have raised important issues in many research fields, such as printed circuit board problems [3] and facility layout problems [4], and effective optimization methods that can avoid the handling of overlapping constraints have been reported [5], [6]. 
However, these methods have limitations, such as the degradation of solutions when resolving overlap, and application that is restricted to partitioning problems or problems that use identical shapes. On the other hand, sequence-pair representation can avoid overlaps among components and allow the manipulation of various sizes of rectangles [7]. Several papers report that this representation enables very effective layout optimization in packing-type problems [8] and facility layout problems [9]. Therefore, sequence-pair representation is used in the proposed new layout optimization method for RCMS.

The main difference between RCMS layout problems and conventional packing-type or general facility layout problems is that optimal spacing among distributed components is extremely important in RCMS problems. This paper proposes a dummy component approach to provide especially appropriate spacing between a single robot and other components. In the following section, the design requirements for the RCMS are clarified first and then quantitative design criteria are formulated. Next, a sequence-pair representation scheme for layout optimization is introduced and an optimization procedure is proposed. Finally, the proposed optimization method is applied to numerical examples to demonstrate the effectiveness of the proposed method.

\section{Criteria for layout design}

\subsection{Layout design problem}

The most important consideration when developing new manufacturing systems is operation time, however, feasibility verification of assembly tasks is also an essential process at the RCMS layout design stage. Another highly important consideration is the minimization of layout area, to enhance the efficiency of factory-level layouts. The quantitative criteria for these requirements are discussed in the following subsections.

\subsection{Productivity}

In this paper we evaluate robot motion time based on the rotational angle of robot arm joints between starting and terminal points for each robot motion. Since the maximum angular velocity value of each assembly robot joint is usually given at the layout design stage, we approximately calculate the robot motion time by assuming that the joint motion has a uniform velocity based on its maximum angular velocity. Hence, the robot motion time $T_{k}^{i}$ of the $k$-th joint performing the $i$-th motion is given in the following equation:

$$
T_{k}^{i}=\frac{\left|\bar{q}_{k}^{i}\right|}{\omega_{k}}
$$

where $\omega_{k}$ indicates the maximum angular velocity of the $k$-th joint and $\bar{q}_{k}^{i}$ is the angle through which the $k$-th joint moves during the $i$-th motion. We assume that all joints can simultaneously have their angle changed, and the $i$-th motion time is evaluated using the maximum joint motion time, as follows:

$$
T^{i}=\max _{k}\left[\frac{\left|\bar{q}_{k}^{i}\right|}{\omega_{k}}\right]
$$

Therefore, the total robot motion time for one assembly cycle $T$ is evaluated as follows:

$$
T=\sum_{i=1}^{h} T^{i}
$$

where $h$ represents the number of robot motions in one assembly cycle.

\subsection{Manipulability}

Manipulability is a quantitative measure of the manipulating ability of robot arms that can be calculated based on the relationship between the position of the end-effector and joint vectors.

Consider a manufacturing system using an $n$ degree-of-freedom assembly robot which conducts tasks at $h$ positions, with its $k$-th joint variable at the $j$-th task position denoted as $q_{k}^{j}$, with joint vector $\mathbf{q}^{j}$. The robot posture vector $\mathbf{r}^{j}$ at the $j$-th 
position $(j=1,2, \cdots, h)$ is defined as follows:

$$
\begin{gathered}
\mathbf{q}^{j}=\left[q_{1}^{j}, q_{2}^{j}, \cdots, q_{n}^{j}\right]^{\top} \quad(j=1,2, \cdots, h) \\
\mathbf{r}^{j}=\left[r_{1}^{j}, r_{2}^{j}, \cdots, r_{m}^{j}\right]^{\top},
\end{gathered}
$$

where $m$ is the number of dimensions needed to represent the position and angle of the end-effector.

Then, the relationship between $\mathbf{r}^{j}$ and $\mathbf{q}^{\mathrm{j}}$ is represented as follows:

$$
\mathbf{r}^{j}=f\left(\mathbf{q}^{j}\right)
$$

In the following discussion, the notation of task position $\mathrm{j}$ is ignored for simplicity. By the chain-rule, velocity vector $\mathbf{v}(=\dot{\mathbf{r}})$ is obtained as

$$
\mathbf{v}=\mathbf{J}(\mathbf{q}) \dot{\mathbf{q}},
$$

where $\dot{\mathbf{q}}$ indicates the joint velocity vector and $\mathbf{J}(\mathbf{q})$ is the Jacobian. In the case of non-redundant robots, the manipulability $w$ is calculated as follows:

$$
w=|\operatorname{det} \mathbf{J}(\mathbf{q})|
$$

The larger the manipulability $w$, the faster the end-effector can move in any direction, implying that larger manipulability values indicate that the robot can conduct more complicated tasks. We therefore calculate the value of $w$ to evaluate the feasibility of the assembly tasks [10]. Using the superscript of task position $j$, the overall feasibility of assembly task $W$ in a robotic cellular manufacturing system is evaluated using the product of manipulability values at $h$ task positions, as follows:

$$
W=\prod_{j=1}^{h} w^{j},
$$

where $w^{j}$ is the manipulability at the $j$-th task position.

\subsection{Layout area}

In this paper, every manufacturing system component is assumed to be rectangular, and layout area $S$ is evaluated using the area of the smallest rectangle that can contain all allocated components, as shown in Fig. 1.

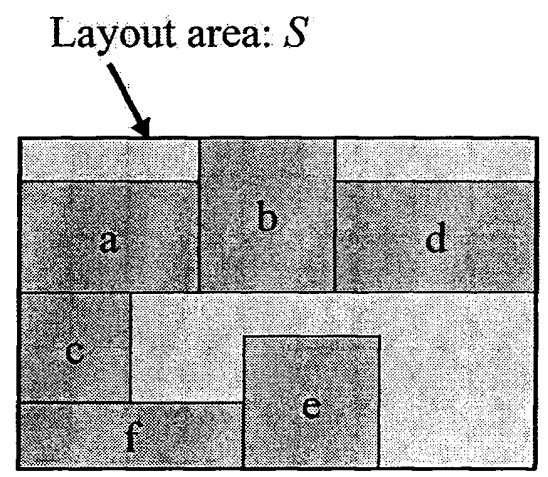

Figure 1: Layout area

\subsection{Optimization problem}

Using the above three design criteria for the RCMS layout, a multi-objective optimization problem for its design can be formulated as follows:

$$
\left\{\begin{array}{cl}
\operatorname{minimize} & T, S \\
\operatorname{maximize} & W
\end{array}\right.
$$




\section{Layout representation scheme}

\subsection{Sequence pair representation}

The sequence-pair representation approach can avoid a constraint resolution process for overlapping constraints, since the native representation inherently prohibits overlaps. The sequence-pair approach employs a pair of sequences, $\Gamma+$ and $\Gamma-$, where each sequence indicates the relative positions of manufacturing system components. Furthermore, in this paper, an additional variable, $\theta_{l}(l=1,2, \ldots, L)$, is introduced to represent the orientation of each allocated rectangular component, where $l$ denotes the component number and $\mathrm{L}$ is the total number of components. Since we assume that all components are rectangular, their orientation, i.e., long side vertical or long side horizontal, is represented by $\theta_{l} \in\{0,1\}$.

\subsection{Representation of distance between components}

In the sequence-pair approach, the space between components is not considered. However, RCMS layout design problems must consider the inclusion of adequate space between components, since robot manipulation requires a certain minimal distance between assembly robot and task points to allow complex assembly tasks to be conducted at these points.

This paper proposes the use of dummy components to represent the spaces in RCMS layout problems that are needed in order to carry out task operations. Several rectangular dummy components are introduced in an optimal layout design problem, and the layout of both ordinary and dummy components is simultaneously optimized. Consequently, dummy components are allocated at places where space is required, allowing layout optimization considering the spacing requirements of the employed robots. If unnecessary dummy components are included in the result of a layout optimization problem, such components are allocated to the outside of the layout domain. Fig. 2 shows an example of a layout including dummy components. In this figure, component $a, b, c, d, e$, and $f$ are ordinary, and $g, h$, and $i$ are dummy.

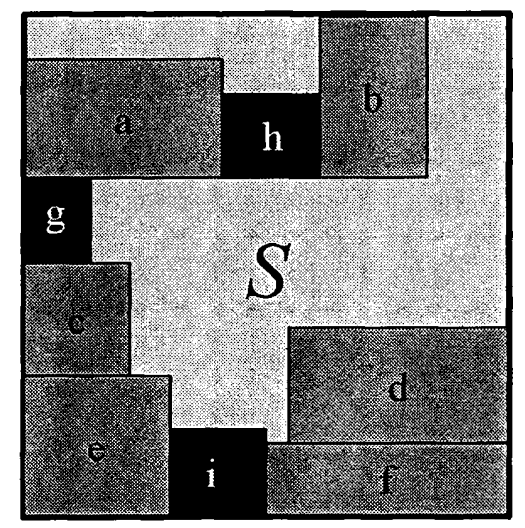

Figure 2: Layout with dummy components

\section{Optimization method}

\subsection{Genetic algorithm}

The proposed layout optimization problem has permutation variables that represent the relative position of components, and binary variables that represent the orientation of rectangular components. The use of meta-heuristic approaches such as genetic algorithms (GAs) [11], particle swarm optimization [12], simulated annealing [13], and an ant system [14] are effective for solving large-scale combinatorial optimization problems, and a further advantage of GAs is that they can provide non-dominated solutions for multi-objective optimization problems. Therefore, this paper uses a GA to solve RCMS layout problems, and the following sections describe its crossover, mutation and selection operators.

\subsection{Crossover}

This paper utilizes Placement-based Partially Exchanging Crossover (PPEX) [15]. In PPEX, a pair of individuals is first chosen, a component in one individual of the pair is then selected at random, and a window domain is created around this component. The sequence and orientation of components inside this window is exchanged with those in the other individual. An example of the PPEX procedure is described in Figs. 3 and 4. 


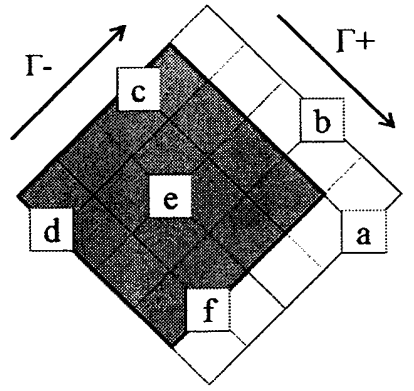

Figure 3: PPEX window
Parent 1

c d e b flald felclabll 0.0 .100

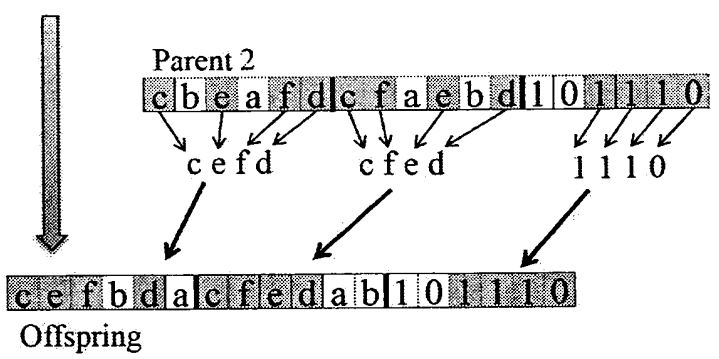

Figure 4: PPEX manipulation

\subsection{Mutation}

A mutation operator is applied to a component at a preassigned mutation probability. When the mutation operator acts on a component, another component in the same individual is chosen at random. The sequence of these two components in permutations $\Gamma+$ and $\Gamma-$ are then exchanged and the orientation of $\theta_{l}$ of these two components is changed.

\subsection{Selection}

The layout design optimization problem proposed in this paper is a multi-objective optimization problem regarding robot motion time, manipulability, and layout area. In this paper, a Non-dominated Sorting Genetic Algorithm (NSGA)-II [16], a multi-objective optimization technique based on a GA, is used. NSGA-II can provide a non-dominated solution set for a multi-objective optimization problem in a single computation, and the obtained non-dominated solution set provides trade-off information among the objective functions.

In the NSGA-II selection scheme, non-dominated solutions are preserved and carried over into the next generation, and a crowdedness criterion is used to select non-dominated solutions existing in sparsely distributed areas, to obtain widely distributed non-dominated solutions.

\section{Numerical example}

\subsection{Problem settings}

The proposed RCMS layout design method is now applied to an example problem. As an example problem, we consider the following sequential tasks:

(1) Robot 1 picks up a part from Part box 1.

(2) Robot 1 changes the pose of the part and passes it to Robot 2.

(3) Robot 2 changes the pose of the part again and passes it to Robot 3.

(4) Robot 3 places the part in Part box 5.

The purpose of these jobs is to change the attitude of assembly parts and line them up so that they can be assembled together during later processes.

The assembly robot used in the following problem is shown in Fig. 5 and Table 1 provides layout component information, i.e., the dimensions and quantity of the components. The number of individuals, the terminal generation number, crossover rate, and mutation rate are set to $100,10000,0.8$ and $1 / 2 L$, respectively, where $L$ is the number of components, including dummy components. The optimization calculation was conducted five times for each problem setting, and only the non-dominated solutions of the five optimization calculations are shown in the figures.

From previous studies, dummy components having various edge lengths are effective when the most appropriate size is unknown. Therefore 12 rectangular dummy components of various sizes, shown as Machine ID 6 17, are used. In this example, we consider robot motion time, manipulability, and layout area as objective functions. 


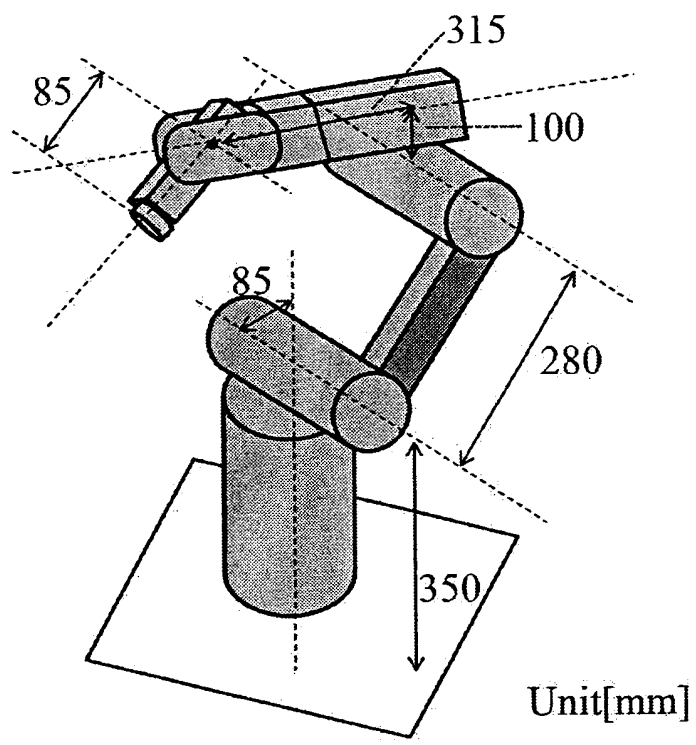

Figure 5: Assembly robot (RV-6SQ)

Table 1: Cellular manufacturing system layout components

\begin{tabular}{|c|c|c|}
\hline Machine ID (name) & $\begin{array}{c}\text { Size [mm] } \\
\text { (height, width) }\end{array}$ & Quantity \\
\hline $\mathbf{1}$ (Robot 1) & $(255,255)$ & 1 \\
\hline $\mathbf{2}$ (Robot 2) & $(255,255)$ & 1 \\
\hline $\mathbf{3}$ (Robot 3) & $(255,255)$ & 1 \\
\hline $\mathbf{4}$ (Part box 1) & $(150,100)$ & 1 \\
\hline $\mathbf{5}$ (Part box 2) & $(150,100)$ & 2 \\
\hline $\mathbf{6 , 7}$ (Dummy component 1) & $(200,190)$ & 2 \\
\hline $\mathbf{8 , 9}$ (Dummy component 2) & $(180,170)$ & 2 \\
\hline $\mathbf{1 0 , 1 1}$ (Dummy component 3) & $(160,150)$ & 2 \\
\hline $\mathbf{1 2 , 1 3}$ (Dummy component 4) & $(140,130)$ & 2 \\
\hline $\mathbf{1 4 , 1 5}$ (Dummy component 5) & $(120,110)$ & 2 \\
\hline $\mathbf{1 6 , 1 7}$ (Dummy component 6) & $(100,90)$ & 1 \\
\hline
\end{tabular}




\subsection{Results}

Using the above parameters and components, the three-objective layout optimization problem is solved in order to verify its validity. The distribution of the obtained non-dominated solutions is shown in Fig. 6, where trade-off relationships among the three objective functions can be observed. The trade-off relationship between layout area and manipulability is especially significant, as shown in Fig. 7.

The layouts of the four solutions indicated by black squares in Figs. 6 and 7 are illustrated in Figs. 8 to 11. Figure 8 shows the layout of solution $\mathrm{A}$, which has the largest manipulability and the largest layout area among the non-dominated solutions. On the other hand, Figure 9 shows the layout of compromise solution B, which has the smallest manipulability and the smallest layout area. Figure 10 shows the layout of compromise solution $\mathrm{C}$, which has the shortest cycle time. Figure 11 shows the layout of compromise solution $\mathrm{D}$, which has reasonable performance values for every criterion.

These figures also imply the existence of a trade-off relationship between layout area and manipulability. That is to say, a smaller layout area reduces manipulability since the robot arm must undergo more radical movements during assembly operations. Thus, an adequate layout area is necessary to obtain sufficient manipulability.

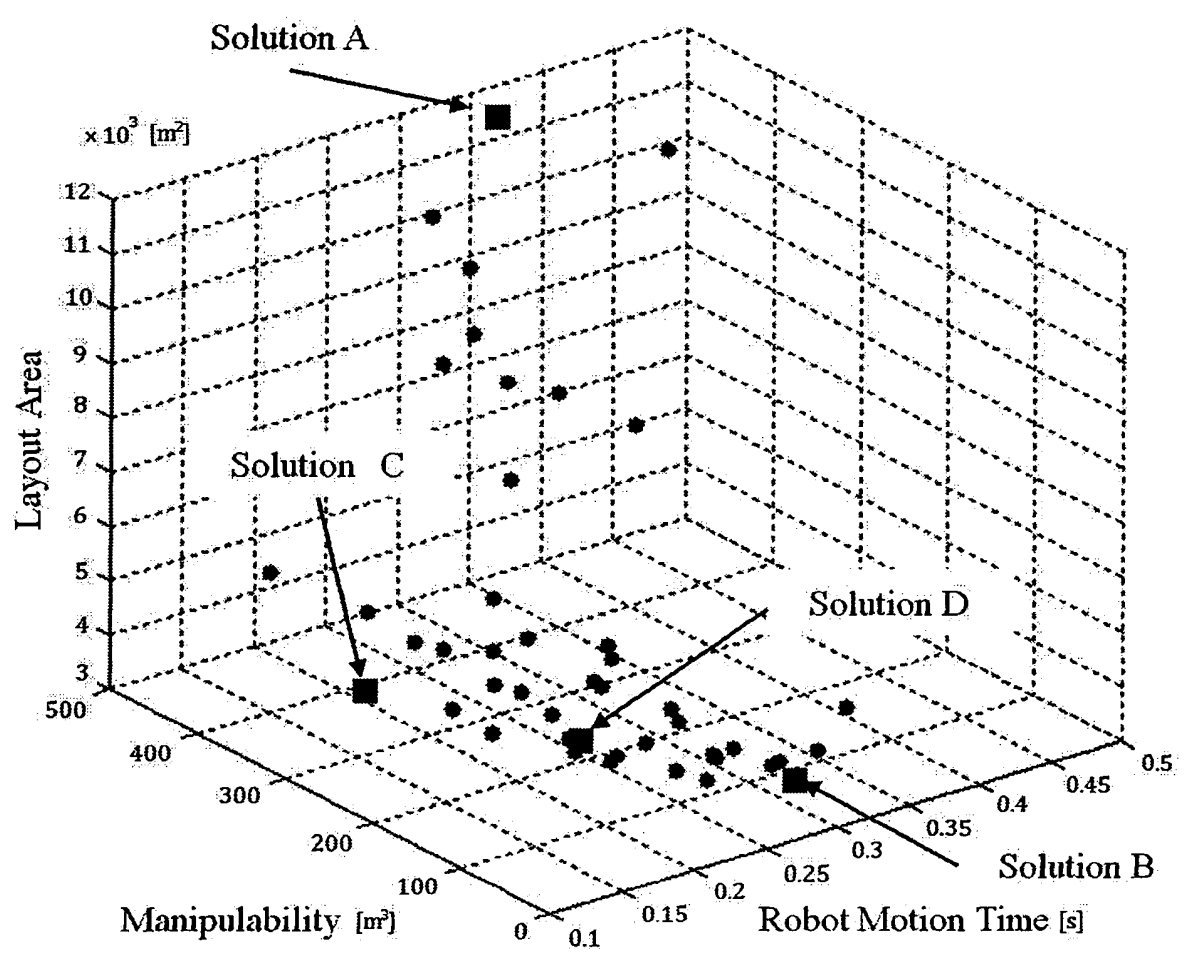

Figure 6: Non-dominated solutions 


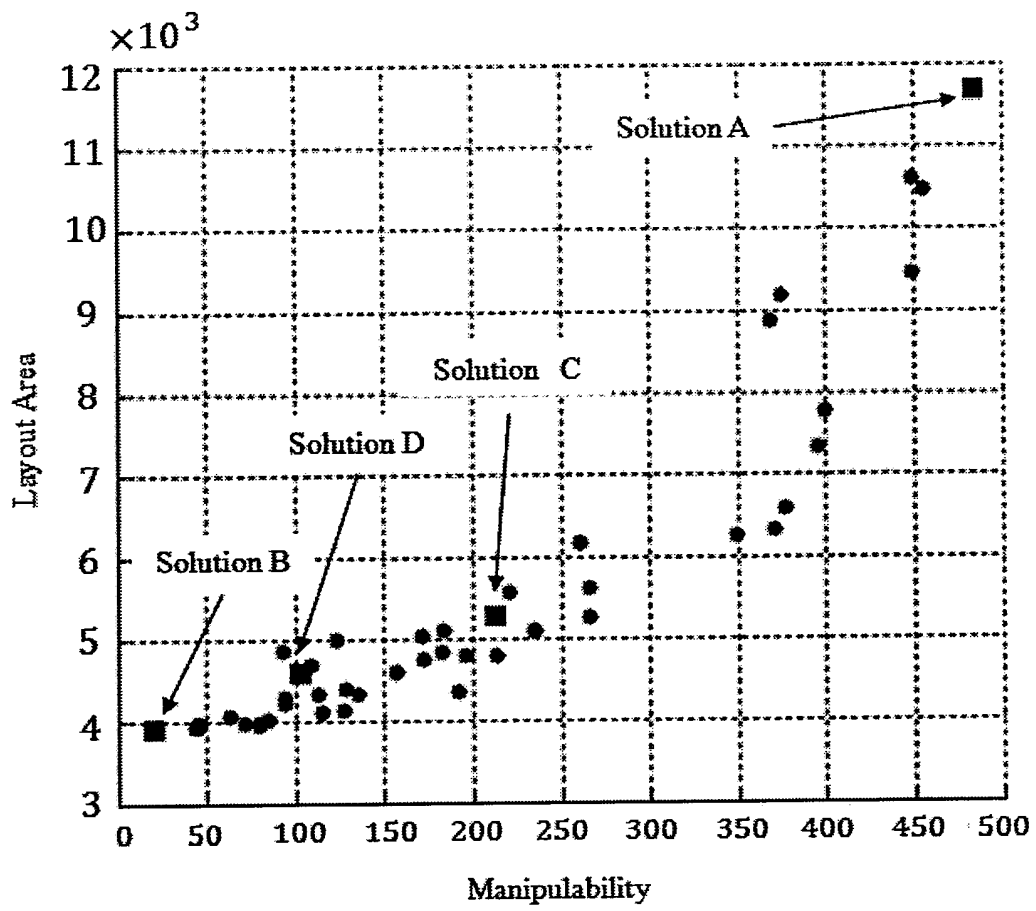

Figure 7: Non-dominated solutions mapped to the Layout area-Manipulability plane

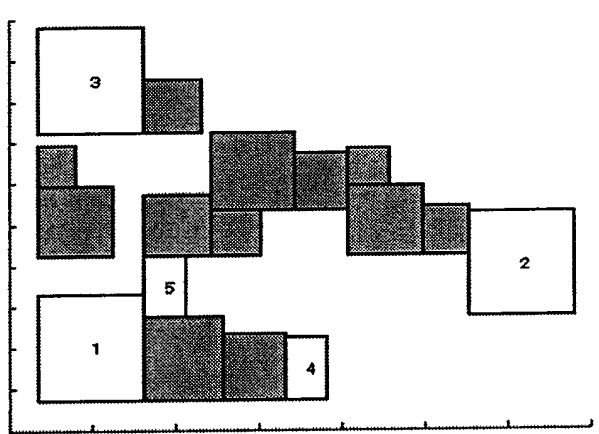

Figure 8: Solution A

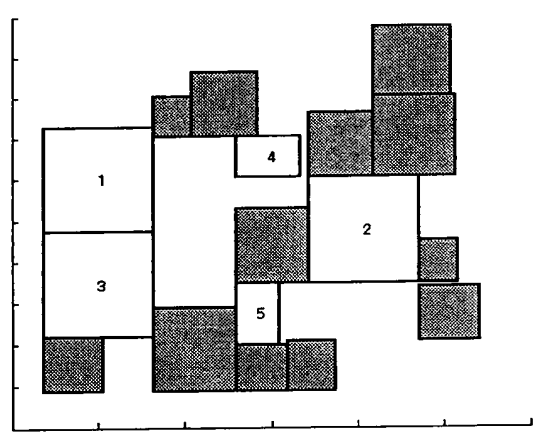

Figure 10: Solution C

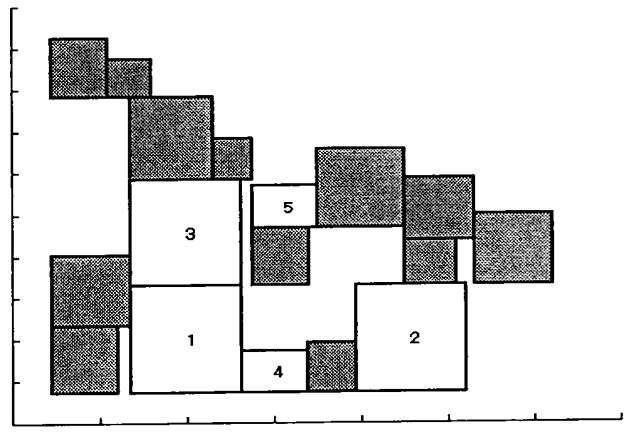

Figure 9: Solution B

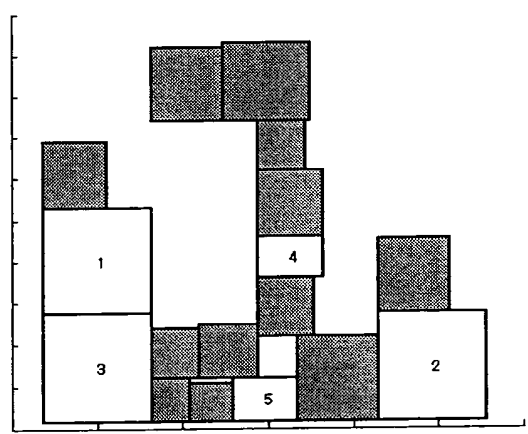

Figure 11: Solution D 


\section{Conclusions}

This paper proposed a new layout optimization method for RCMS including cooperative tasks between three robots. First, the design requirements for RCMS layouts were clarified. Robot motion time, manipulability, and layout area were proposed as design criteria and an optimization problem was formulated. A sequence-pair scheme was introduced for the RCMS layout representation, to avoid overlapping among assembly system components during the optimization procedure. Furthermore, the use of dummy components was proposed, to enhance obtaining solutions that have optimal spacing between components. A multi-objective genetic algorithm was used to solve the multi-objective combinatorial optimization problems. A PPEX-type operator was used for crossover of sequence-pair chromosomes and NSGA-II was used to obtain non-dominated solutions. The proposed method was applied to numerical examples, and its effectiveness was demonstrated.

\section{References}

[1] Barral, D., Perrin, J. P., Dombre, E., \& Liegeois, A., Simulated annealing combined with a constructive algorithm for optimizing assembly workcell Layout, The International Journal of Advanced Manufacturing Technology, 17 (1), 593-602, 2001.

[2] Tay, M. L. \& Ngoi, B. K. A., Optimizing robot workcell layout, The International Journal of Advanced Manufacturing Technology, 12 (5), 377-385, 1996.

[3] Shahookar, K. \& Mazumder, P., VLSI cell placement techniques, ACM computing surveys, 23 (2), 143-220, 1991.

[4] Gen, M., Lin, L., \& Zhang, H., Evolutionary techniques for optimization problems in integrated manufacturing system: State-of-the-art-survey, Computers \& Industrial Engineering, 56 (3), 779-808, 2009.

[5] Chen, T.-C., \& Chang, Y.-W., Modern floorplanning based on $\mathrm{B}^{\mathrm{L}}$-tree fast simulated annealing, IEEE Transactions on Computer-Aided Design of Integrated Circuits and Systems, 25 (4), 637-650, 2006.

[6] Birgin, E. G, \& Lobato, R. D., Orthogonal packing of identical rectangles within isotropic convex regions, Computers \& Industrial Engineering, 59 (4), 595-602, 2010.

[7] Murata, H., Fujiyoshi, K., Nakatake, S., \& Kajitani, Y., VLSI module placement based on rectangle packing by the sequence-pair, IEEE Transactions on Computer-Aided Design of Integrated Circuits and Systems, 15 (12), 1518-1524, 1996.

[8] Drakidis, A., Mack, R. J., \& Massara, R. E., Packing-based VLSI Module Placement Using Genetic Algorithm with Sequence-pair Representation, IEE Proceedings - Circuits, Devices \& Systems, 156 (6), 545-551, 2006.

[9] Meller, R. D., Chen, W. P., \& Sherali, H. D., Applying the sequence-pair representation optimal facility layout designs, Operations Research Letters, 35(5), 651-659, 2007.

[10] Yoshikawa, T., Manipulability of robotic mechanisms, International Journal of Robotics Research, 4 (2), 3-9, 1985.

[11] Solimanpur, M. \& Kamran, M. A., Solving facilities location problem in the presence of alternative processing routes using a genetic algorithm, Computers \& Industrial Engineering, 59 (4), 830-839, 2010.

[12] Samarghandi, H., Taabayan, P. \& Jahantigh, F. F., A particle swarm optimization for the single row facility layout problem, Computers \& Industrial Engineering, 58(4), 529-534, 2010.

[13] Sahin, R., Ertogral, K., \& Turkbey, O., A simulated annealing heuristic for the dynamic layout problem with budget constraint, Computers \& Industrial Engineering, 59 (2), 308-313, 2010.

[14] Komarudin \& Wong, K. Y., Applying ant system for solving unequal area facility layout problems, European Journal of Operational Research, 202 (3), 730-746, 2010.

[15] Nakaya, S., Wakabahashi, S., \& Koide, T., An adaptive genetic algorithm with sequence-pair representation of solutions for VLSI floorplanning, The 2000 IEEE International Symposium on Circuits and Systems, 3 , 65-68, 2000.

[16] Deb, K., Pratap, A., Agarwal, S., \& Meyarivan, T., A fast and elitist multiobjective genetic algorithm: NSGA-II, IEEE Transaction Evolutionary Computation, 6 (2), 182-197, 2002. 